\title{
Visual Face Perception of Adults With Unilateral Cleft Lip and Palate in Comparison to Controls-An Eye-Tracking Study
}

\author{
Philipp Meyer-Marcotty, D.D.S., Ph.D., Antje B.M. Gerdes, Ph.D., Angelika Stellzig-Eisenhauer, D.D.S., Ph.D., \\ Georg W. Alpers, Ph.D.
}

\begin{abstract}
Objective: To assess how faces with a cleft lip and palate are perceived and to study how faces with and without a unilateral cleft lip and palate are viewed by individuals with a unilateral cleft lip and/or palate in comparison to nonaffected controls.

Design: Prospective clinical study.

Setting: Department of Orthodontics and Department of Psychology, University of Wuerzburg.

Participants: Thirty-three participants (20 men and 13 women; mean age, 25.4 \pm 6.6 years) with a unilateral cleft lip and/or palate and a control group of 30 participants (15 men and 15 women; mean age, $26.8 \pm 3.4$ years) were enrolled in this study.

Main Outcome Measures: Eye movements were analyzed via an eye-tracking camera while all participants looked at pictures of faces with and without a unilateral cleft lip and palate.

Results: The nose and the mouth area of pictures of faces with a unilateral cleft lip and palate were looked at significantly longer by both groups. Additionally, the participants with a unilateral cleft lip and/or palate looked at faces with and without a unilateral cleft lip and palate differently, taking more time to view the nose and less time to view the eyes compared with the participants without a cleft lip and palate.

Conclusion: When perceiving a face with a unilateral cleft lip and palate, the observer's gaze is distracted to the nose and mouth area. Moreover, participants with a unilateral cleft lip and/or palate themselves focused greater attention on those features that are anomalous on their own faces in comparison to participants without a cleft lip and palate. Specifically, this different scanpath is reflected in the cumulative duration of the eye movements as well as in the initial facial scan pattern.
\end{abstract}

KEY WORDS: asymmetry, cleft lip and palate, eye tracking, scanpath

The human face is the most important stimulus in social interaction. The impact of attractiveness on social communication has been widely acknowledged (Watson and Thornhill, 1994; Rhodes, 2006). Accordingly, the literature describes that patients with facial malformations suffer significant disadvantages in their social lives. Previous studies found higher levels of behavioral inhibition in

Dr. Meyer-Marcotty is Lecturer, Department of Orthodontics, Dental Clinic of the Medical Faculty; Dr. Gerdes is Lecturer, Department of Psychology; and Dr. Stellzig-Eisenhauer is Chair and Professor, Department of Orthodontics, Dental Clinic of the Medical Faculty, University of Wuerzburg, Wuerzburg, Germany. Dr. Alpers is Professor, Department of Psychology, University of Wuerzburg, Wuerzburg, Germany, and Professor, Department of Psychology, University of Eichstaett, Eichstaett, Germany.

Submitted October 2008; Accepted June 2010.

Address correspondence to: Dr. Philipp Meyer-Marcotty, Department of Orthodontics, Dental Clinic of the Medical Faculty, Pleicherwall 2, D97070 Wuerzburg, Germany. E-mail Meyer_P1@klinik.uni-wuerzburg.de.

DOI: $10.1597 / 08-244$ patients exhibiting a deformed appearance and a higher prevalence of depression and anxiety (Thompson and Kent, 2001).

The social consequences of facial disfigurement suggest that patients with cleft lip and palate (CLP) are at a disadvantage in social life (Hunt et al., 2005). The facial appearance of patients with a unilateral CLP (UCLP) may be seriously affected in two very important regions of the face: the nose and the mouth. Even when CLP repair is completed in early infancy and followed up by interdisciplinary therapeutic rehabilitation, a residual scar on the upper lip and a distinct asymmetry of the nose usually remain into adulthood. For this reason, it can be conjectured that these slight but visible abnormalities may evoke impairments in social functioning.

Supporting this assumption is the high incidence of teasing due to facial appearance reported among children with a CLP (Hunt et al., 2006). Moreover, individuals with a CLP have been found to be affected by social isolation and poor self-esteem (Lockhart, 2003). Contradictory 
results were reported about the psychosocial effects of the CLP (Hunt et al., 2005). The literature has tended to report the psychosocial functioning of patients with a CLP in a general way. Psychosocial problems do not occur at a general level in the majority of patients with a CLP, although some specific problems may arise in relation to interpersonal relationships (e.g., difficulties arising in social situations with unknown people and/or with unknown expectations [Hunt et al., 2005; Brand et al., 2009]).

Recent studies have focused on the question of satisfaction and dissatisfaction with facial appearance in adult patients with a CLP (Marcusson et al., 2002; Sinko et al., 2005). A high degree of dissatisfaction $(>50 \%)$ and a desire for further treatment $(>60 \%)$ in adults was found after the treatment of patients with a CLP (Marcusson et al., 2002; Sinko et al., 2005). In this context it would be interesting to know whether the faces of patients with a CLP are perceived differently than nonaffected faces. Furthermore, the question arises as to how individuals with a CL/P themselves look at faces with and without a CLP. However, up to now no objective data have existed in the literature that analyze how faces of patients with a CLP are viewed.

A sophisticated approach to studying the visual perception of faces involves analyzing eye movements. The eyes are constantly moving as they scan and inspect visual scenes. Eye movements play an important role in visual perception, and analyzing them can provide information about the process of perception (Noton and Stark, 1971). The analysis of eye movements via an eye-tracking camera has been used in numerous fields of psychological research, which confirms the reproducibility and the validity of the method (Rayner, 1998; Alpers, 2008). Visual perception during natural viewing is characterized by a gaze-controlled sampling strategy (Mertens et al., 1993). This means that the eyes alternate between fixations, when they are aimed at a fixed point in the visual field, and saccades, the rapid eye movements that lead to a new fixed point on the area of interest. Noton and Stark (1971) demonstrated that the temporal and spatial sequence of fixations and saccades is not randomly distributed.

When looking at a face in particular, the eyes usually fixate on the main features. The eyes follow a regular pathway from feature to feature in a fixed order, the socalled scanpath (Noton and Stark, 1971). While looking at a face with a neutral expression, the typical scanpath is a top-down movement with primary fixation on the eyes, followed by the nose and mouth (Yarbus, 1967; Walker Smith et al., 1977; Althoff and Cohen, 1999; Loughland et al., 2002; Whalen et al., 2004; Eisenbarth and Alpers, 2006; Yuki et al., 2007). In recent studies, the visual perception of faces was analyzed by tracking subjects' scanpath with a camera in an experimental setting (Mertens et al., 1993; Althoff and Cohen, 1999; Lundqvist et al., 1999). It was shown that the internal features of the face are particularly important in face processing and that there is a left-visualfield advantage in the recognition of faces (Mertens et al.,
1993). Previous work with eye tracking provides wellfounded documentation that viewers initially fixate on emotionally salient features of a visual display and for longer durations than less salient features. Thus, the pattern of fixation can be interpreted as an objective psychophysiological marker of visual attention. We have documented this when healthy individuals examine two photographs (one emotionally relevant, one neutral) (Alpers, 2007) or when phobic patients pursue a visual search task involving phobic material (Gerdes et al., 2008). However, whether some or all of these effects are initially or intentionally controlled remains controversial (Moors and De Houwer, 2006).

In any case, we expect that the CLP-related features of the face will be more emotionally salient to persons with a $\mathrm{CL} / \mathrm{P}$ and that this will result in preferential allocation of attention to these features.

Although there is considerable evidence that individuals with a CL/P suffer from the psychosocial consequences of their facial appearance in spite of advanced maxillofacial surgery, there are no data on how their faces are perceived by others and how persons with a CL/P perceive faces with a cleft. Therefore, the aims of this study were (1) to examine whether faces of adults with a UCLP are viewed with a different scanpath than faces of a control group without congenital anomalies; and (2) to examine how participants with a UCL/P look at faces with and without a UCLP in contrast to participants without a CLP.

Our hypothesis was that participants with a UCL/P would be characterized with a different scanpath than nonaffected participants while looking at faces with a UCLP and that these differences could be quantified using eye-movement recordings. A focus of attention of participants with UCL/P on CLP-related features (the nose and mouth region) was hypothesized.

\section{MethodS}

\section{Patients/Participants}

Thirty-three adult participants with a UCL/P (20 men and 13 women) were recruited from the Department of Orthodontics of the University of Wuerzburg, Wuerzburg, Germany. The mean age of the participants was $25.4 \pm$ 6.6 years. Twenty-four participants had a complete UCLP, and six had a complete unilateral cleft lip (UCL). Inclusion criteria for entering the study were (1) normal vision and (2) no other congenital facial anomalies or other distinctive facial features such as piercing or tattoos.

The control group consisted of 30 participants (15 women and 15 men; mean age, $26.8 \pm 3.4$ years). They were selected via an announcement in the newspaper. Inclusion criteria were (1) normal vision, (2) no current medical or dental treatment, and (3) no congenital facial anomaly or other distinctive facial features such as piercing or tattoos. There was no significant difference between the 


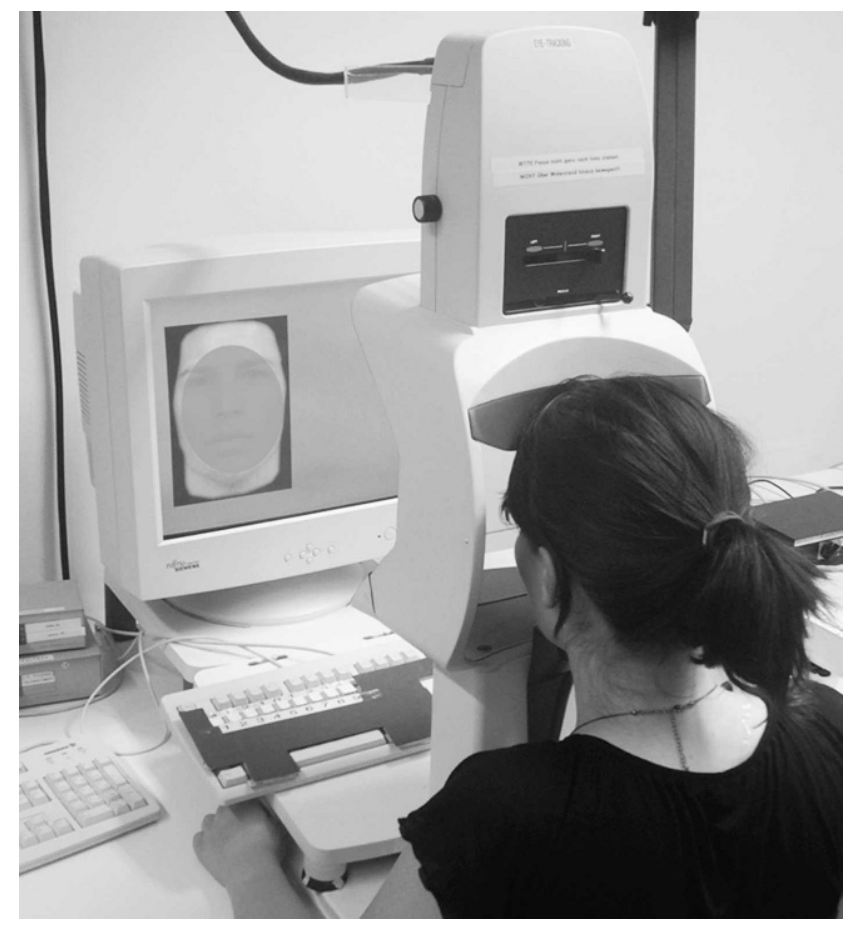

FIGURE 1 Experimental set-up used in this study. Recording of the eye movements with an infrared camera while participants view pictures of the faces on a screen. For anonymity, the entire face on the screen was masked.

patients and the participants according to age $\left(\mathrm{t}_{61}=.26, p\right.$ $=.79)$ and gender $\left(\mathrm{t}_{61}=.84 ; p=.41\right)$.

\section{Picture Material}

Black-and-white pictures of 18 adults with a UCLP (nine women and nine men; mean age, 21.6 years) and 18 controls (nine women and nine men) age-matched to the CLP group were presented to all participants. In all photos, the subject had a neutral facial expression and eyes looking straight ahead. All pictures were taken against the same dark background and were masked beneath the chin and around the head, so that ears, hair, and other peripheral features were eliminated. The picture size was set to $412 \times$ 581 pixels with a resolution of 96 pixels per inch. All pictures were presented twice, once in the original orientation and once mirrored in order to avoid any bias between right and left sides. A total of 72 pictures were shown.

\section{Apparatus}

All 63 participants were seated comfortably at a distance of $50 \mathrm{~cm}$ in front of a 17 -inch monitor (resolution $=1024 \times$ 768) (Fig. 1); for anonymity, the entire face on the screen was masked. The head was stabilized by a chin and a forehead support in order to eliminate head movements (for a more detailed description, see Alpers, 2008; Gerdes et al., 2008). Picture material presentation was controlled by the software program Presentation ${ }^{\circledR}$ (version 0.90; Neuro- behavioral Systems, Inc., Albany, NY; http://www.neurobs. com).

Eye movements were recorded with an iView $\mathrm{X}^{\mathrm{TM}} \mathrm{Hi}-$ Speed camera (SensoMotoric Instruments, Teltow, Germany). The system is a video-based device that uses an infrared camera to capture eye movements. Images of the eye's position were sampled at $238 \mathrm{~Hz}$ and a spatial resolution of approximately $0.5^{\circ}$ to $1^{\circ}$.

\section{Procedure-Eye Tracking}

The eye tracker was calibrated for each participant's right eye using an 11-point calibration procedure. This is the procedure with the highest accuracy within the iView $\mathrm{X}^{\mathrm{TM}}$ Hi-Speed 1250 system (SensoMotoric; Gerdes et al., 2008). Therefore, the specified features of a tracking resolution of $<0.01^{\circ}$ and a gaze-position accuracy of $0.25^{\circ}$ to $0.5^{\circ}$ could be attained.

The eye movements of the participants were recorded continuously while they viewed the pictures of the faces. The task was introduced with on-screen instructions and four practice trials. They were told that they would see a series of different faces and that they were allowed to look at them in any manner that they wished.

Before each picture was presented, a fixation cross was presented in the center of the screen. After the fixation cross was continuously fixated for 1.5 seconds, the picture material was presented in random order. The picture material appeared either to the left or the right side of the computer screen in order to release a first saccade toward the presented face. Each picture was shown for 5 seconds. The intertrial interval was 250 milliseconds.

This study was independently reviewed and approved by the institutional review board at the Medical Faculty of the University of Wuerzburg (study reference number of the ethics committee: 177/07). The experiments were undertaken with the understanding and written consent of each participant according to principles of the Declaration of Helsinki.

\section{Data Analysis}

Fixations were defined as scanpath data limited to a maximum radius of $2.02^{\circ}$ visual angle for at least 80 milliseconds (BEGAZE Software; SensoMotoric) (see Alpers, 2008). For the analysis of the eye-movement data, the main morphological areas for face perception (eyes, nose, and mouth) were defined as areas of interest (AOIs) (Mertens et al., 1993). The definition of the AOIs was in accordance with the anthropometric landmarks (eyes: exocanthion to endocanthion; nose: subnasale to nasion; mouth: sublabiale to subnasale). The number and duration of fixations on these AOIs were examined.

Furthermore, the fast fixation sequence of the first three fixations was investigated as an index of initial attentional capture, and the cumulative duration of all fixations on 
TABLE 1 Mean of the First Three Fixations (in \%) of the Participants With a UCL/P and Participants Without a CLP for the AOI: (1) Eyes, (2) Nose, and (3) Mouth According to the Picture Categories (UCLP Versus Control)* ${ }^{*}$

\begin{tabular}{llccc}
\hline \multirow{2}{*}{ AOI } & $\begin{array}{c}\text { Picture } \\
\text { Category }\end{array}$ & $\begin{array}{c}\text { Participants With } \\
\text { a UCL/P, Mean } \\
(S D) \dagger\end{array}$ & $\begin{array}{c}\text { Participants } \\
\text { Without a CLP, } \\
\text { Mean }(S D)\end{array}$ & $p$ Value \\
\hline \multirow{2}{*}{ Eyes } & UCLP & $37.98(10.83)$ & $45.69(13.09)$ & .013 \\
& Control & $40.61(12.89)$ & $48.59(13.08)$ & .018 \\
Nose & UCLP & $53.93(9.47)$ & $45.42(10.20)$ & .001 \\
& Control & $52.86(10.16)$ & $45.00(11.18)$ & .005 \\
Mouth & UCLP & $8.08(6.28)$ & $8.88(7.20)$ & .64 \\
& Control & $6.52(5.85)$ & $6.41(5.87)$ & .94 \\
\hline
\end{tabular}

* $\mathrm{UCL} / \mathrm{P}=$ unilateral cleft lip and/or palate; $\mathrm{CLP}=$ cleft lip and palate; $\mathrm{AOI}=$ area of interest; UCLP = unilateral cleft lip and palate.

$\dagger$ Participants with a UCL/P, $\mathrm{n}=33$; participants without a CLP, $\mathrm{n}=30 ; t$ test.

each AOI was investigated as an index of sustained attentional engagement. Both indices result in the scanpath, defined as the eye-movement pattern. Analyses of variance (ANOVAs) were made with these eye-movement parameters as dependent variables. The factors were group (participants with a UCL/P versus participants without a CLP), picture category (CLP versus control), and AOI (eyes, nose, mouth). For all analyses, the alpha level was set at .05. Significant effects were followed-up by $t$ tests (twotailed).

\section{Results}

\section{Initial Attentional Capture}

In order to examine the initial attentional capture of the participants with a UCL/P versus the participants without a CLP, the mean (in percentages) of the first three fixations was investigated in both groups on the three AOIs (eyes, nose, mouth) for both picture categories (UCLP versus control).

The ANOVA for the mean percentage of the first three fixations revealed a significant main effect of AOI $\left(\mathrm{F}_{2,122}=\right.$ 245.66, $p<.001)$. The main effects of picture category and group were not significant $(p>.05)$. A significant AOI by group interaction $\left(\mathrm{F}_{2,122}=6.36, p=.007\right)$ and a significant AOI by picture category interaction $\left(\mathrm{F}_{2,122}=213.56, p<\right.$ $.001)$ were analyzed. No significant effect of group by picture category interaction was found $(p>.05)$. There was a significant AOI by picture category by group interaction $\left(\mathrm{F}_{2,122}=7.17, p=.004\right)$.

Separate ANOVAs for both picture categories showed a significant effect of AOI $\left(\mathrm{F}_{2,122}=217.07, p<.001\right)$ and a significant $\mathrm{AOI}$ by group interaction in the control pictures $\left(\mathrm{F}_{2,122}=6.24, p=.009\right)$. In UCLP pictures, a significant effect of AOI $\left(\mathrm{F}_{2,122}=211.27, p<.001\right)$ and a significant AOI by group interaction $\left(\mathrm{F}_{2,122}=7.31, p=.003\right)$ could be found.

The follow-up $t$ test for the mean percentage of the first three fixations showed significant differences between participants with a UCL/P and participants without a
TABLE 2 Mean of the Cumulative Duration of All Fixations (in ms) of Both Groups (Participants With a CL/P and Participants Without a CLP, $N=63$ ) for the AOI: (1) All AOI Summarized and Each AOI Separately: (2) Eyes, (3) Nose, and (4) Mouth According to the Picture Categories (UCLP Versus Control)*

\begin{tabular}{lccc}
\hline \multicolumn{1}{c}{ AOI } & $\begin{array}{c}\text { UCLP Pictures, } \\
\text { Mean }(S D) \dagger\end{array}$ & $\begin{array}{c}\text { Control Pictures, } \\
\text { Mean }(S D)\end{array}$ & $p$ Value \\
\hline All AOI & $677.95(130.16)$ & $646.43(120.32)$ & $<.001$ \\
Eyes & $895.66(251.53)$ & $935.25(269.39)$ & .045 \\
Nose & $820.75(284.55)$ & $751.37(276.27)$ & $<.001$ \\
Mouth & $321.96(142.89)$ & $254.56(127.79)$ & $<.001$ \\
\hline
\end{tabular}

$* \mathrm{CL} / \mathrm{P}=$ cleft lip and/or palate; CLP $=$ cleft lip and palate; $\mathrm{AOI}=$ area of interest; $\mathrm{UCLP}=$ unilateral cleft lip and palate.

$\dagger t$ test.

CLP in both picture categories for the AOIs eyes and nose (Table 1).

Participants with a UCL/P fixated on the eyes significantly less frequently than did the participants without a CLP for pictures of faces with a UCLP $\left(\mathrm{t}_{61}=2.55, p=\right.$ $.013)$ and control pictures $\left(\mathrm{t}_{61}=2.47, p=.018\right)$. In contrast, participants with a UCL/P fixated on the nose significantly more often than did the participants without a CLP for the pictures of faces with UCLP $\left(\mathrm{t}_{61}=3.42, p=\right.$ $.001)$ and the control pictures $\left(\mathrm{t}_{61}=2.93, p=.005\right)$.

No differences were found between the two groups for the fixations on the AOI mouth in both picture categories.

In summary, a significant difference in the initial attentional capture between participants with a UCL/P and participants without a CLP was observable in the perception of faces with UCLP and control pictures in the first three fixations. This demonstrated a different fixation pattern among participants with a UCL/P, who exhibited more fixations on the nose and fewer fixations on the eyes.

\section{Sustained Attentional Engagement}

To compare the sustained attentional engagement with pictures of faces with UCLP and control pictures, the cumulative duration of all fixations of both groups was examined.

The ANOVA of the cumulative duration of all fixations indicated a significant main effect of picture category $\left(\mathrm{F}_{1,60}\right.$ $=24.37, p<.001)$ and a main effect of AOI $\left(\mathrm{F}_{2,120}=\right.$ $163.59, p<.001)$. No significant effect of group was found $(p>.05)$. There was a significant effect of picture category by AOI interaction $\left(\mathrm{F}_{2,120}=11.16, p<.001\right)$ and a significant AOI by group interaction $\left(\mathrm{F}_{2,120}=12.15, p<\right.$ $.001)$. No significant interaction of group by picture category was found $(p>.05)$.

Follow-up $t$ tests showed that pictures of faces with a UCLP were looked at significantly longer than the control pictures independently by group (by summarizing all three AOIs as "all AOIs") ( $\left.\mathrm{t}_{62}=4.83, p<.001\right)$ (Table 2).

Additionally, in the analysis of each AOI, it was shown that when pictures of faces with a UCLP were viewed, the cumulative duration of all fixations on the eyes was 
TABLE 3 Mean of the Cumulative Duration of All Fixations (in ms) of the Participants With a UCL/P and Participants Without a CLP for the AOI: (1) Eyes, (2) Nose, and (3) Mouth According to the Picture Categories (UCLP Versus Control) $* \dagger$

\begin{tabular}{llccc}
\hline \multirow{2}{*}{ AOI } & $\begin{array}{c}\text { Picture } \\
\text { Category }\end{array}$ & $\begin{array}{c}\text { Participants With } \\
\text { a CL/P, Mean }(S D)\end{array}$ & $\begin{array}{c}\text { Participants Without } \\
\text { a CLP, Mean }(S D)\end{array}$ & $p$ Value \\
\hline \multirow{2}{*}{ Eyes } & UCLP & $821.60(197.52)$ & $977.12(281.26)$ & .015 \\
& Control & $827.96(203.33)$ & $1053.27(286.40)$ & .001 \\
\multirow{2}{*}{ Nose } & UCLP & $899.73(317.15)$ & $733.87(217.20)$ & .020 \\
& Control & $838.82(305.87)$ & $655.17(204.14)$ & .007 \\
\multirow{2}{*}{ Kouth } & UCLP & $324.33(135.57)$ & $309.86(159.05)$ & .70 \\
& Control & $258.63(108.72)$ & $250.22(147.23)$ & .80 \\
\hline
\end{tabular}

${ }^{*} \mathrm{CL} / \mathrm{P}=$ cleft lip and/or palate; CLP $=$ cleft lip and palate; AOI $=$ area of interest; $\mathrm{UCLP}=$ unilateral cleft lip and palate.

$\dagger$ Participants with a CL/P, $\mathrm{n}=33$; participants without a CLP, $\mathrm{n}=30 ; t$ test.

significantly shorter than when control pictures were viewed $\left(\mathrm{t}_{62}=2.05, p=.045\right)$. In contrast, pictures of faces with a UCLP were viewed significantly longer in the nose $\left(\mathrm{t}_{62}=3.82, p<.001\right)$ and mouth area $\left(\mathrm{t}_{61}=5.69, p<.001\right)$ than the control pictures (Table 2).

When analyzing the AOI by group interaction, it was found that the participants with a UCL/P looked a significantly shorter time at the eyes in CLP pictures $\left(\mathrm{t}_{61}\right.$ $=2.52, p=.015)$ and in control pictures $\left(\mathrm{t}_{61}=3.57, p=\right.$ .001) than did the participants without a CLP (Table 3). In contrast, participants with a UCL/P looked at the nose for a significantly longer time than did participants without a CLP when scanning both pictures of faces with a UCLP $\left(\mathrm{t}_{61}\right.$ $=2.40, p=.020)$ and control pictures $\left(\mathrm{t}_{61}=2.77, p=\right.$ .007). No differences were found between the two groups for the mouth (Table 3).

Taken together, the results of the sustained attentional engagement showed first that both groups of participants looked at pictures of faces with a UCLP with a longer cumulative duration of all fixations than at pictures of faces without a UCLP, in particular, with a longer view of the nose and the mouth. Second, the results showed that participants with a UCL/P compared with the participants without a CLP looked at the nose for a longer period and at the eyes for a shorter period when scanning pictures of faces with a CLP as well as faces without a CLP.

\section{DisCUSSION}

We performed an eye-tracking study because there were no data available on how individuals with a UCLP are looked at by others and how participants with a UCL/P themselves look at pictures of individuals with a CLP and at individuals without a CLP. The method involved measuring the eye movements of adults with a UCL/P and participants without a CLP. While recording the eye movements, pictures of faces with UCLP and control pictures without an anomaly were presented. The main findings of the study were that faces of individuals with a UCLP are looked at differently compared with nonaffected faces and that participants with a UCL/P themselves looked at faces differently than did participants without a CLP.
The mean percentage of the first three fixations, used as an index of initial attentional capture, and the cumulative duration of all fixations, used as an index of sustained attentional engagement, were analyzed in all participants. Both indices quantify the scanpath, which is defined as the eye-movement pattern while looking at faces. For this reason, the scanpath was analyzed in participants with a $\mathrm{UCL} / \mathrm{P}$ and in nonaffected participants.

The results of our study show that participants with a UCL/P had a significantly different scanpath during the initial attentional capture with more fixations on the nose and fewer fixations on the eyes than did the participants without a CLP. The varying pattern of eye movements was independent of the picture category (CLP pictures versus control pictures). This could be interpreted as a more sensitive scan pattern in the initial perception of the face. The fact that participants with a UCL/P focus more on the nose area might be explained by their own experience of having an anomaly in this area. Therefore, the primary hypothesis of a focus of attention of participants with a $\mathrm{UCL} / \mathrm{P}$ on CLP-related features (the nose and mouth region) appears to be confirmed.

These are the first data to report on face perception in individuals with a UCL/P. To obtain initial eye-tracking data there was no differentiation between the subgroups CLP and CL. Both subgroups were defined as a single group with a visible defect. In the literature it is not unusual to combine both groups for analyzing different aspects of intersocial behavior and facial appearance (Thomas et al., 1997; Hunt et al., 2006). Nevertheless, in future studies it would be interesting to analyze face perception according to cleft type.

The sustained attentional engagement with the pictures of UCLP faces showed that they were looked at for a significantly longer cumulative duration of all fixations and, in particular, that both groups gazed at the nose and mouth for a longer period of time. The results indicate that the scanpath is distracted to the asymmetric areas of the face in UCLP pictures. Therefore, it could be suggested that the asymmetry caused by a CLP may be one decisive factor for aberrant visual perception when looking at a face with a UCLP.

Moreover, the participants with a UCL/P showed different cumulative duration of all fixations compared with the participants without a CLP. Participants with a $\mathrm{UCL} / \mathrm{P}$ looked at the nose area for a longer period and at the area of the eyes for a shorter period in both picture categories; whereas, the participants without a CLP looked at the area of the eyes for a longer period and at the nose area for a shorter period. Therefore, it could be suggested that participants with a UCL/P are characterized by a different scanpath than nonaffected participants when looking at faces. We interpret the group differences that we found in fixations as indications of a preferential allocation of attention to CLP-relevant facial features in participants with a UCL/P. Most likely, this indicates that 
CLP-relevant facial features are more salient to participants with a UCL/P. One explanation may be that this reflects the negative emotional connotation of these facial features for participants with a UCL/P.

Furthermore, this result implies the particular importance of the nose in participants with a UCL/P. The nose is undoubtedly the most prominent facial feature, which is in plain view and impossible to cover or hide (Babuccu et al., 2003). An asymmetry in this important area of the face could influence the attractiveness of the patients and consequently their satisfaction with their facial appearance. Therefore, many adults (more than 50\%) with a UCLP express dissatisfaction with their facial appearance after completion of their growth (Richman, 1976; Kapp, 1979; Thomas et al., 1997; Marcusson et al., 2002) and desire further treatment, of the nose in particular, to improve their facial appearance (Marcusson et al., 2002). An aberration from normal symmetric morphology in this part of the face was shown to be the most likely feature to be teased about in childhood (Semb et al., 2005). Thus, facial asymmetry in patients with a CLP may be a reason for social and emotional distress. This experience of teasing in childhood (Hunt et al., 2006) and the increased dissatisfaction with their facial appearance as adults (Meyer-Marcotty and Stellzig-Eisenhauer, 2009) may explain the different behavior in direct face-to-face interaction in adults with UCL/P.

Additionally, social inhibition or shyness has been identified repeatedly as one behavioral characteristic in patients with a CLP (Endriga and Kapp-Simon, 1999). This finding could be supported by the analysis of the eyemovement pattern in participants with a UCL/P, as found in our study. A lower frequency of looking in the eyes in face-to-face interaction of participants with a UCL/P compared with participants without a CLP could increase the impression of shyness in social interaction.

In this study a previously neglected topic was investigated. Therefore, the aim of this study was not to test a hypothesis but to examine experimentally the visual perception of faces with or without CLP. Given that the study was exploratory, the use of Bonferroni corrections was felt to be too conservative (Miller, 1981). To confirm the present results, further studies need to be carried out. Moreover, in future research, the etiology of the different scan pattern in individuals with a CL/P should be analyzed with functional magnetic resonance imaging. This would enable examination of any neurological or neurobiological differences that could result in social dysfunction (Nopoulos et al., 2005).

One limitation of this study may be the fact that we presented still photographs for an extended period of time, a method often used in the study of facial expressions (see Adolph and Alpers, in press). Our study participants may have reacted differently to the pictures on the screen than they would to faces in real social encounters. Thus, our index of prolonged attentional engagement may indicate only how faces with UCLP are inspected when the viewers do not feel bound to the rules of social interactions. However, it is not at all unusual to start out such research with well-controlled picture material and then proceed to more realistic but more difficult to control real-life observations later. Moreover, typical behavioral effects of social interactions relevant for everyday interactions such as mimicry have also been observed in studies using still photographs (Dimberg et al., 2002; Neumann et al., 2005; Eisenbarth and Alpers, 2006).

\section{Conclusion}

Participants with a UCL/P and participants without a CLP looked at faces of individuals with a UCLP differently compared with the way they viewed nonaffected faces. Fixations of the observers' eyes were more frequently on the nose and mouth area in UCLP faces.

Additionally, participants with a UCL/P themselves focused greater attention on those features that are anomalous on their own faces in comparison with participants without a CLP. When they view faces of patients with a UCLP and faces without an anomaly, they gaze at the nose for a longer period of time and at the eyes for a shorter period. Specifically, this different scanpath is reflected in the voluntary control of the eye movements as well as in the initial facial scan pattern.

\section{REFERENCES}

Adolph D, Alpers GW. Valence and arousal: a comparison of two sets of emotional facial expressions. Am J Psychol. 2010;123:209-219.

Alpers GW. Eye-catching: right hemisphere attentional bias for emotional pictures. Laterality. 2008;13:158-178.

Alpers GW, Gerdes ABM. Here's looking at you: emotional faces predominate in binocular rivalry. Emotion. 2007;7:495-506.

Althoff RR, Cohen NJ. Eye-movement-based memory effect: a reprocessing effect in face perception. J Exp Psychol Learn Mem Cogn. 1999;25:997-1010.

Babuccu O, Latifoglu O, Atabay K, Oral N, Cosan B. Sociological aspects of rhinoplasty. Aesthet Plast Surg. 2003;27:44-49.

Brand S, Blechschmidt A, Müller A, Sader R, Schwenzer-Zimmerer K, Zeilhofer HF, Holsboer-Trachsler E. Psychosocial functioning and sleep patterns in children and adolescents with cleft lip and palate (CLP) compared with healthy controls. Cleft Palate Craniofac J. 2009;46:124-135.

Corbetta M, Akbudak E, Conturo TE, Snyder AZ, Ollinger JM, Drury HA, Linenweber MR, Petersen SE, Raichle ME, Van Essen DC, et al. A common network of functional areas for attention and eye movements. Neuron. 1998;21:761-773.

Dimberg U, Thunberg M, Grunedal S. Facial reactions to emotional stimuli: automatically controlled emotional responses. Cogn Emot. 2002;16:449-471.

Eisenbarth H, Alpers GW. Eyes and mouth: competing for attention in emotional faces. J Psychophysiol. 2006;20:130.

Endriga MC, Kapp-Simon KA. Psychological issues in craniofacial care: state of the art. Cleft Palate Craniofac J. 1999;36:3-11.

Gerdes ABM, Alpers GW, Pauli P. When spiders appear suddenly: spiderphobic patients are distracted by task-irrelevant spiders. Behav Res Ther. 2008;46:174-187. 
Hunt O, Burden D, Hepper P, Johnston C. The psychosocial effects of cleft lip and palate: a systematic review. Eur J Orthod. 2005;27:274-285.

Hunt O, Burden D, Hepper P, Stevenson M, Johnston C. Self-reports of psychosocial functioning among children and young adults with cleft lip and palate. Cleft Palate Craniofac J. 2006;43:598-605.

Kapp K. Self-concept of the cleft lip and/or palate child. Cleft Palate J. 1979;16:171-176.

Lockhart E. The mental health needs of children and adolescents with cleft lip and/or palate. Clin Child Psychol Psychiatry. 2003;8:7-16.

Loughland CM, Williams LM, Gordon E. Visual scanpaths to positive and negative facial emotions in an outpatient schizophrenia sample. Schizophr Res. 2002;55:159-170.

Lundqvist D, Esteves F, Öhman A. The face of wrath: critical features for conveying facial threat. Cogn Emot. 1999;13:691-711.

Marcusson A, Paulin G, Ostrup L. Facial appearance in adults who had cleft lip and palate treated in childhood. Scand J Plast Reconstr Surg Hand Surg. 2002;36:16-23.

Mertens I, Siegmund H, Grüsser OJ. Gaze motor asymmetries in the perception of faces during a memory task. Neuropsychologia. 1993;31: 989-998.

Meyer-Marcotty P, Stellzig-Eisenhauer A. Dentofacial self-perception and social perception of adults with unilateral cleft lip and palate. J Orofac Orthop. 2009;70:224-236.

Miller RG. Simultaneous Statistical Inference. 2nd ed. New York: Springer-Verlag; 1981.

Moors A, De Houwer J. Automaticity: a theoretical and conceptual analysis. Psychol Bull. 2006;132:297-326.

Neumann R, Hess M, Schulz S, Alpers GW. Automatic behavioural responses to valence: evidence that facial action is facilitated by evaluative processing. Cogn Emot. 2005;19:499-513.

Nopoulos P, Choe I, Berg S, Van Demark D, Canady J, Richman L. Ventral frontal cortex morphology in adult males with isolated orofacial clefts: relationship to abnormalities in social function. Cleft Palate Craniofac J. 2005;42:138-144.
Noton D, Stark I. Eye movements and visual perception. Sci Am. 1971;224:35-43.

Rayner K. Eye movements in reading and information processing: 20 years of research. Psychol Bull. 1998;124:372-422.

Rhodes G. The evolutionary psychology of facial beauty. Annu Rev Psychol. 2006;57:199-226.

Richman LC. Behavior and achievement of cleft palate children. Cleft Palate J. 1976;13:4-10.

Semb G, Brattström V, Mølsted K, Prahl-Andersen B, Zuurbier P, Rumsey N, Shaw WC. The Eurocleft study: intercenter study of treatment outcome in patients with complete cleft lip and palate. Part 4: relationship among treatment outcome, patient/parent satisfaction, and the burden of care. Cleft Palate Craniofac J. 2005;42:83-92.

Sinko K, Jagsch R, Prechtl V, Watzinger F, Hollmann K, Baumann A. Evaluation of esthetic, functional, and quality-of-life outcome in adult cleft lip and palate patients. Cleft Palate Craniofac J. 2005;42:355-361.

Thomas PT, Turner SR, Rumsey N, Dowell T, Sandy JR. Satisfaction with facial appearance among subjects affected by a cleft. Cleft Palate Craniofac J. 1997;34:226-231.

Thompson A, Kent G. Adjusting to disfigurement: processes involved in dealing with being visibly different. Clin Psychol Rev. 2001;21:663-682.

Walker Smith GJ, Gale AG, Findlay JM. Eye movement strategies involved in face perception. Perception. 1977;6:313-326.

Watson PM, Thornhill R. Fluctuating asymmetry and sexual selection. Trends Ecol Evol. 1994;9:21-25.

Whalen PJ, Kagan J, Cook RG, Davis FC, Kim H, Polis S, McLaren DG, Somerville LH, McLean AA, Maxwell JS, et al. Human amygdala responsivity to masked fearful eye whites. Science. 2004;306:2061.

Yarbus AL. Eye Movements and Vision. New York: Plenum; 1967.

Yuki M, Maddux WW, Masuda T. Are the windows to the soul the same in the East and West? Cultural differences in using the eyes and mouth as cues to recognize emotions in Japan and the United States. $J$ Exp Soc Psychol. 2007;43:303-311. 